\title{
Prolongation structures for supersymmetric equations
}

\author{
G H M Roelofs and N W van den Hijligenberg \\ Department of Applied Mathematics, University of Twente, PO Box 217, $7500 \mathrm{AE}$ \\ Enschede, The Netherlands
}

Received 25 June 1990

\begin{abstract}
The well known prolongation technique of Wahlquist and Estabrook for nonlinear evolution equations is generalized for supersymmetric equations and applied to the supersymmetric extension of the KdV equation of Manin-Radul. Using the theory of Kac-Moody Lie superalgebras, the explicit form of the resulting Lie superalgebra is determined. It is shown to be isomorphic to $R_{\mathrm{MR}} \times \mathrm{Cov}^{+}(C(2), \sigma)$, where $R_{M R}$ is an eight dimensional radical. An auto-Bäcklund transformation is derived from the prolongation structure and the relationship with known solution methods of the SKdV equation is analysed. In addition it is indicated how a superposition principle for the SKdV equation can be obtained.
\end{abstract}

\section{Introduction}

In their famous article [1] Wahlquist and Estabrook developed prolongation theory for nonlinear evolution equations and applied it to the Korteweg-de Vries (KdV) equation. This resulted in a Lie algebra structure, which has been determined explicitly by both Estabrook [2] and van Eck [3]. Article [4] is an example of how the theory of KacMoody algebras can be used fruitfully to obtain a realization of a prolongation algebra. Wahlquist and Estabrook showed the relationship between the existence of non-trivial prolongation structures and known solution methods for nonlinear evolution equations such as associated inverse scattering problems and Bäcklund transformations, indicating the relevance of the method.

In this article we will generalize prolongation theory to the case of supersymmetric evolution equations and apply it to the SKdV equation of Manin-Radul (MR). Successively we realize the resulting Lie superalgebra using the theory of Kac-Moody Lie superalgebras, derive an auto-Bäcklund transformation from the prolongation structure and indicate how to obtain a superposition principle for the SKdV equation. Moreover, we analyse the relationship between the super prolongation method and known solution methods for the SKdV equation such as the super-Miura transformation and the super-Gardner transformation.

The paper is organized as follows. In section 2 we give a short summary of graded differential geometry in order to fix notation and to facilitate working with graded vector fields and differential forms and derive a generalization of the prolongation method of Wahlquist and Estabrook for supersymmetric evolution equations. In section 3 we apply this method to the SKdV equation and realize the resulting Lie superalgebra. Finally in section 4 we derive an auto-Bäcklund transformation and the superposition principle for the SKdV equation from the prolongation structure and analyse the relationship with known solution methods of the SKdV equation. 


\section{Prolongation method for supersymmetric systems}

In this section we give a generalization of Wahlquist and Estabrook's prolongation method. Before that we first give a short summary of graded differential geometry [1].

\subsection{Graded differential geometry}

In order to fix notation and to facilitate working with graded vector fields and graded differential forms in the subsequent sections of the paper, we give a short summary of the local picture of a graded manifold $X$ of dimension $(m, n)$ in this section. For a more detailed description we refer the reader to Kostant [5].

In the following we denote by $M_{k}$ the set of multi-indices $I=\left(i_{1}, \ldots, i_{k}\right)$ which satisfy $1 \leq i_{1}<\cdots<i_{k} \leq m$ and by $N_{l}$ the set of multi-indices $\nu=\left(\nu_{1}, \ldots, \nu_{n}\right)$ with $\nu_{i} \in \mathbb{N}$ and $\sum \nu_{i}=$ l. Furthermore, for each $\mathbb{Z}_{2}$-graded vector space $A$ we denote by $A_{i}$ the subspace of homogeneous elements of degree $i \in \mathbb{Z}_{2}$. We define $\Lambda(n)$ as the exterior algebra on $n$ generators $s_{1}, \ldots, s_{n}$. With this definition $\Lambda(n)$ becomes a commutative superalgebra. As a set of local odd coordinates of $X$ we take the generators of $\Lambda(n)$, the even coordinates can be taken as in the case of an ordinary smooth manifold of dimension $m$.

Smooth functions on this graded manifold can locally be seen as elements of the commutative superalgebra $A(U) \equiv C^{\infty}(U) \otimes_{\mathbb{R}} \Lambda(n)$, where $U$ is an open subset in $\mathbb{R}^{m}$ and $C^{\infty}(U)$ is the commutative algebra of smooth real-valued functions on $U$. Each element $f$ in $A(U)$ can be represented as

$$
f=\sum_{k=0}^{n} \sum_{\mu \in M_{k}} f_{\mu} \otimes s_{\mu}
$$

with $f_{\mu} \in C^{\infty}(U)$ and $s_{\mu}=s_{\mu_{1}} s_{\mu_{2}} \cdots s_{\mu_{k}}$. It is obvious that the elements $\sum_{k \bmod 2=i} \sum_{\mu \in M_{k}} f_{\mu} \otimes s_{\mu}$ span the homogeneous space of degree $i \in \mathbb{Z}_{2}$.

Graded vector fields and graded differential forms can locally be described in terms of specific objects concerning the commutative superalgebra $A(U)$. Graded vector fields are elements of the space Der $A(U)$ of graded derivations on $A(U)$. One can show that $\operatorname{Der} A(U)$ is a free left $A(U)$ module with basis $\left\{\partial_{x_{i}}, \partial_{s_{j}} \mid 1 \leq i \leq m, 1 \leq j \leq n\right\}$, i.e. each $\theta \in \operatorname{Der} A(U)$ can be written as

$$
\theta=\sum_{i=1}^{m} f_{i} \partial_{x_{1}}+\sum_{j=1}^{n} g_{j} \partial_{s}, \quad\left(f_{i}, g_{j} \in A(U)\right) .
$$

The derivations $\partial_{x_{1}} \in(\operatorname{Der} A(U))_{0}$ and $\partial_{s} \in(\operatorname{Der} A(U))_{1}$ are defined by

$$
\partial_{x_{\imath}} f=\sum_{k=0}^{n} \sum_{\mu \in M_{k}} \partial_{x_{1}} f_{\mu} \otimes s_{\mu} \quad \partial_{s_{j}} f=\sum_{k=0}^{n} \sum_{\mu \in M_{k}} f_{\mu} \otimes \partial_{s},\left(1 \otimes s_{\mu}\right)
$$

where $\partial_{s,}\left(1 \otimes s_{k}\right)=\delta_{j, k}$. It is obvious that $\theta \in(\operatorname{Der} A(U))_{k}$ if and only if $f_{i} \in A(U)_{k}$ and $g_{j} \in A(U)_{k+1}$ in the representation above. Der $A(U)$ is a Lie superalgebra with commutator

$\left[\theta_{1}, \theta_{2}\right] \equiv \theta_{1} \circ \theta_{2}-(-1)^{i j} \theta_{2} \circ \theta_{1} \quad\left(\theta_{1} \in(\operatorname{Der} A(U))_{i}, \theta_{2} \in(\operatorname{Der} A(U))_{j}\right)$ 
For later reference we give this commutator for the case $m=n=1$ explicitly. For $f^{0} \in A(U)_{k}, g^{0} \in A(U)_{l}, f^{1} \in A(U)_{k+1}$ and $g^{1} \in A(U)_{l+1}$ we have

$$
\begin{gathered}
{\left[f^{0} \partial_{x}+f^{1} \partial_{s}, g^{0} \partial_{x}+g^{1} \partial_{s}\right]=\left\{f^{0} \partial_{x} g^{0}-(-1)^{k l} g^{0} \partial_{x} f^{0}+f^{1} \partial_{s} g^{0}-(-1)^{k l} g^{1} \partial_{s} f^{0}\right\} \partial_{x}} \\
+\left\{f^{0} \partial_{x} g^{1}-(-1)^{k l} g^{0} \partial_{x} f^{1}+f^{1} \partial_{s} g^{1}-(-1)^{k l} g^{1} \partial_{s} f^{1}\right\} \partial_{s}
\end{gathered}
$$

Graded differential $k$-forms are defined as elements of the space $\Omega^{k}(A, U)$ of all $k$ - $A(U)$-linear maps $\beta$ on $\operatorname{Der} A(U)$ which for all $1 \leq i<k, \theta_{i} \in(\operatorname{Der} A(U))_{k}$ and $\theta_{i+1} \in(\operatorname{Der} A(U))_{l}$ satisfy

$$
\left\langle\theta_{1}, \ldots, \theta_{i}, \theta_{i+1}, \ldots, \theta_{k} \mid \beta\right\rangle=-(-1)^{k l}\left\langle\theta_{1}, \ldots, \theta_{i+1}, \theta_{i}, \ldots, \theta_{k} \mid \beta\right\rangle
$$

As in the ordinary case we can introduce a multiplication $\wedge$ of graded differential forms. This turns $\Omega(A, U) \boxminus \bigoplus_{k \in \mathbb{N}} \Omega^{k}(A, U)$ into a bigraded commutative algebra. Thus, for each $\beta \in\left(\Omega^{k}(A, U)\right)_{i}, \gamma \in\left(\Omega^{l}(A, U)\right)_{j}$ we have

$$
\beta \wedge \gamma=(-1)^{k l+i j} \gamma \wedge \beta
$$

If we define $\mathrm{d} x_{i} \in\left(\Omega^{1}(A, U)\right)_{0}$ and $\mathrm{d} s_{j} \in\left(\Omega^{1}(A, U)\right)_{1}$ in the usual way, one can prove that $\left\{\mathrm{d} x_{I} \wedge \mathrm{d} s^{\nu} \mid I \in M_{k}, \nu \in N_{l}, k+l=p\right\}$ is a basis of $\Omega^{p}(A, U)$ as a free right $A(U)$ module, where

$$
\begin{aligned}
& \mathrm{d} x_{I}=\mathrm{d} x_{i_{1}} \wedge \cdots \wedge \mathrm{d} x_{i_{k}} \\
& \mathrm{~d} s^{\nu}=\mathrm{d} s_{1}^{\nu_{1}} \wedge \cdots \wedge \mathrm{d} s_{n}^{\nu_{n}} \quad \text { with } \mathrm{d} s_{j}^{p}=\underbrace{\mathrm{d} s_{j} \wedge \cdots \wedge \mathrm{d} s_{j}}_{p \text { times }}
\end{aligned}
$$

i.e. each $\beta \in \Omega^{p}(A, U)$ can be written as

$$
\beta=\sum_{k+I=p} \sum_{\substack{I \in M_{k} \\ \nu \in N_{l}}} \mathrm{~d} x_{I} \wedge \mathrm{d} s^{\nu} f_{I, \nu} \quad\left(f_{I, \nu} \in A(U)\right)
$$

Finally we remark that there is a unique operator $\mathrm{d}: \Omega(A, U) \rightarrow \Omega(A, U)$ of degree $(1,0)$ that satisfies $\mathrm{d}^{2}=0$ and

$$
\mathrm{d}(f)=\sum_{i=1}^{m} \mathrm{~d} x_{i} \partial_{x_{i}}(f)+\sum_{j=1}^{n} \mathrm{~d} s_{j} \partial_{s,}(f) \quad\left(f \in \Omega^{0}(A, U)=A(U)\right) .
$$

This operator is the exterior differential operator for graded differential forms. On $\Omega^{p}(A, U)$ it acts as

$$
\mathrm{d} \beta=\sum_{k+l=p} \sum_{\substack{I \in M_{k} \\ \nu \in N_{l}}}(-1)^{p} \mathrm{~d} x_{I} \wedge \mathrm{d} s^{\nu} \wedge \mathrm{d} f_{I, \nu}
$$




\subsection{Generalization of the prolongation method}

In this subsection we give a generalization for systems of supersymmetric evolution equations of the prolongation method described for ordinary evolution equations by Wahlquist and Estabrook [1]. For this purpose we first give a short summary of the prolongation method for an ordinary evolution equation

$$
u_{t}=f\left(u^{(n)}\right)
$$

where $f\left(u^{(n)}\right)$ is a function of $u$ and derivatives of $u$ up to order $n$. Working on the $(n-1)$ th order jet bundle, where we denote the $i$ th order derivative of $u$ by $u_{i}$ (in practical problems we also use the notation $u, u_{x}, u_{x x}, \ldots$ ), we can represent this differential equation by the set of differential 2 -forms

$$
\alpha_{i}=\mathrm{d} u_{i-1} \wedge \mathrm{d} t-u_{i} \mathrm{~d} x \wedge \mathrm{d} t \quad \text { for } i=1, \ldots, n-1
$$

and one additional form $\alpha_{n}$ representing the differential equation. By this we mean that the pullback of these forms to a solution manifold of equation (4) vanishes identically, and reversely, any $(x, t)$-dependent solution $u$ to the set of equations

$$
\alpha_{1}=\cdots=\alpha_{n}=0
$$

satisfies equation (4). From Cartan's theory of differential forms [6] we have an integrability condition on the set of equations (5), namely, if $I=I\left(\alpha_{1}, \ldots, \alpha_{n}\right)$ is the ideal generated by the $\alpha_{i}$ in the exterior algebra with basis $\left\{\mathrm{d} x, \mathrm{~d} t, \mathrm{~d} u, \mathrm{~d} u_{1}, \ldots, \mathrm{d} u_{n-1}\right\}$, we must have that $\mathrm{d} I \subset I$.

The prolongation method consists of adding an additional 1-form

$$
\omega=\mathrm{d} y+F\left(u^{(n-1)}, y\right) \mathrm{d} x+G\left(u^{(n-1)}, y\right) \mathrm{d} t
$$

to the set $\alpha_{1}, \ldots, \alpha_{n}$ and requiring $\mathrm{d} \omega$ to be element of the extended ideal $I\left(\alpha_{1}, \ldots, \alpha_{n}, \omega\right)$. One can easily see that this can be reformulated as follows: add to the original set of forms a Lie algebra valued form

$$
\omega=\mathrm{d} y+F\left(u^{(n-1)}\right) \mathrm{d} x+G\left(u^{(n-1)}\right) \mathrm{d} t
$$

and require $\omega$ to satisfy the condition $\mathrm{d} \omega+[G, F] \mathrm{d} x \wedge \mathrm{d} t \in I\left(\alpha_{1}, \ldots, \alpha_{n}\right)$. This condition leads to an overdetermined system of partial differential equations for $F$ and $G$, which gives rise to a Lie algebra structure to be determined.

In general a set of supersymmetric evolution equations will be of the form

$$
\begin{aligned}
& u_{t}=f\left(u^{(n)}, \varphi^{(m)}\right) \\
& \varphi_{t}=g\left(u^{(n)}, \varphi^{(m)}\right)
\end{aligned}
$$

where $u$ is an even and $\varphi$ is an odd function. Working on an appropriate (graded) jet bundle we can represent this set of equations by the the set of even differential 2-forms

$$
\alpha_{i}=\mathrm{d} t \wedge \mathrm{d} u_{i-1}+\mathrm{d} x \wedge \mathrm{d} t u_{i} \quad \text { for } i=1, \ldots, n-1
$$


and an additional even form $\alpha_{n}^{\prime}$ representing equation (6), together with the set of odd differential 2-forms

$$
\beta_{j}=\mathrm{d} t \wedge \mathrm{d} \varphi_{j-1}+\mathrm{d} x \wedge \mathrm{d} t \varphi_{j} \quad \text { for } j=1, \ldots, m-1
$$

and an additional odd form $\beta_{m}$ representing equation (7). At this place we assume the analogue of Cartan's integrability condition for ordinary differential forms to hold also for graded differential forms, namely $\mathrm{d} I \subset I$ where $I=I\left(\alpha_{1}, \ldots, \alpha_{n}, \beta_{1}, \ldots, \beta_{m}\right)$ is the ideal generated by the $\alpha_{i}$ and $\beta_{j}$ in the (graded) exterior algebra with basis $\left\{\mathrm{d} x, \mathrm{~d} t, \mathrm{~d} u, \ldots, \mathrm{d} u_{n-1}, \mathrm{~d} \varphi, \ldots, \mathrm{d} \varphi_{m-1}\right\}$.

Since in this case we are dealing with even as well as odd differential forms, our generalization of the prolongation method of Wahlquist and Estabrook consists of adding both an even differential 1 -form

$$
\omega^{0}=\mathrm{d} y^{0}+\mathrm{d} x F^{0}\left(u^{(n-1)}, \varphi^{(m-1)}, y^{0}, y^{1}\right)+\mathrm{d} t G^{0}\left(u^{(n-1)}, \varphi^{(m-1)}, y^{0}, y^{1}\right)
$$

and an odd differential 1-form

$$
\omega^{1}=\mathrm{d} y^{1}+\mathrm{d} x F^{1}\left(u^{(n-1)}, \varphi^{(m-1)}, y^{0}, y^{1}\right)+\mathrm{d} t G^{1}\left(u^{(n-1)}, \varphi^{(m-1)}, y^{0}, y^{1}\right)
$$

where $y^{0}$ and $y^{1}$ are a new even and odd variable, respectively, $F^{0}$ and $G^{0}$ are even and $F^{1}$ and $G^{1}$ odd functions, and requiring both $\mathrm{d} \omega^{0}$ and $\mathrm{d} \omega^{1}$ to be element of the extended ideal $I\left(\alpha_{1}, \ldots, \alpha_{n}, \beta_{1}, \ldots, \beta_{m}, \omega^{0}, \omega^{1}\right)$.

Using formulae (2) and (3) we find for $\mathrm{d} \omega^{k}(k=0,1)$

$$
\begin{gathered}
\mathrm{d} \omega^{k}=-\sum_{i=0}^{n-1}\left(\mathrm{~d} x \wedge \mathrm{d} u_{i} F_{u_{i}}^{k}+\mathrm{d} t \wedge \mathrm{d} u_{i} G_{u_{i}}^{k}\right)-\sum_{j=0}^{m-1}\left(\mathrm{~d} x \wedge \mathrm{d} \varphi_{j} F_{\varphi_{j}}^{k}+\mathrm{d} t \wedge \mathrm{d} \varphi_{j} G_{\varphi_{j}}^{k}\right) \\
-\mathrm{d} x \wedge\left(\mathrm{d} y^{0} F_{y^{0}}^{k}+\mathrm{d} y^{1} F_{y^{1}}^{k}\right)-\mathrm{d} t \wedge\left(\mathrm{d} y^{0} G_{y^{0}}^{k}+\mathrm{d} y^{1} G_{y^{1}}^{k}\right) .
\end{gathered}
$$

Using the definition of $\omega^{0}$ and $\omega^{1}$ to eliminate $\mathrm{d} y^{0}$ and $\mathrm{d} y^{1}$ we can rewrite this as

$$
\begin{array}{rl}
\mathrm{d} \omega^{k}=\cdots-\mathrm{d} & x \wedge\left(\omega^{0} F_{y^{0}}^{k}+\omega^{1} F_{y^{1}}^{k}\right)-\mathrm{d} t \wedge\left(\omega^{0} G_{y^{0}}^{k}+\omega^{1} G_{y^{1}}^{k}\right) \\
+\mathrm{d} x & \wedge \mathrm{d} t\left(G^{0} F_{y^{0}}^{k}+G^{1} F_{y^{1}}^{k}-F^{0} G_{y^{0}}^{k}-F^{1} G_{y^{1}}^{k}\right) .
\end{array}
$$

If we define two even vector fields

$$
F=F^{0} \partial_{y^{0}}+F^{1} \partial_{y^{2}} \quad G=G^{0} \partial_{y^{0}}+G^{1} \partial_{y^{1}}
$$

we see from formula (1) that the coefficient of $d x \wedge d t$ in the last expression for $d \omega^{k}$ is the coefficient of $\partial_{y^{k}}$ in $[G, F]$ for $k=0,1$, respectively. Hence $\omega^{0}$ and $\omega^{1}$ can be seen as the components of an even vector field valued form

$$
\omega=\mathrm{d} \tilde{y}+\mathrm{d} x F+\mathrm{d} t G .
$$

As in the ordinary case we can now reformulate the prolongation method as follows: add to the original set of forms an even Lie superalgebra valued form

$$
\omega=\mathrm{d} \bar{y}+\mathrm{d} x F\left(u^{(n-1)}, \varphi^{(m-1)}\right)+\mathrm{d} t G\left(u^{(n-1)}, \varphi^{(m-1)}\right)
$$

and require that $\omega$ satisfy the condition $\mathrm{d} \omega+\mathrm{d} x \wedge \mathrm{d} t[G, F] \in I\left(\alpha_{1}, \ldots, \alpha_{n}, \beta_{1}, \ldots, \beta_{m}\right)$. From the above it is clear that both $F$ and $G$ must be even elements of this Lie superalgebra. 


\section{Application to the SKdV equation}

In this section we apply the method developed in section 2 to the SKdV equation of Manin-Radul

$$
\begin{aligned}
& u_{t}=-u_{x x x}+6 u u_{x}-3 \varphi \varphi_{x x} \\
& \varphi_{t}=-\varphi_{x x x}+3 u \varphi_{x}+3 u_{x} \varphi .
\end{aligned}
$$

In the first subsection we derive the necessary equations and the resulting lie superalgebra, in the second subsection we realize this Lie superalgebra using the theory of Kac-Moody Lie superalgebras.

\subsection{Prolongation of the SKdV equation}

Using the methods described in subsection 2.2 we may represent the SKdV equation by the set of differential 2 -forms

$$
\begin{aligned}
& \alpha_{1}=\mathrm{d} t \wedge \mathrm{d} u+\mathrm{d} x \wedge \mathrm{d} t u_{x} \\
& \alpha_{2}=\mathrm{d} t \wedge \mathrm{d} u_{x}+\mathrm{d} x \wedge \mathrm{d} t u_{x x} \\
& \alpha_{3}=\mathrm{d} t \wedge \mathrm{d} u_{x x}+\mathrm{d} x \wedge \mathrm{d} t\left(6 u u_{x}-3 \varphi \varphi_{x x}\right)-\mathrm{d} x \wedge \mathrm{d} u \\
& \beta_{1}=\mathrm{d} t \wedge \mathrm{d} \varphi+\mathrm{d} x \wedge \mathrm{d} t \varphi_{x} \\
& \beta_{2}=\mathrm{d} t \wedge \mathrm{d} \varphi_{x}+\mathrm{d} x \wedge \mathrm{d} t \varphi_{x x} \\
& \beta_{3}=\mathrm{d} t \wedge \mathrm{d} \varphi_{x x}+\mathrm{d} x \wedge \mathrm{d} t\left(3 u \varphi_{x}+3 u_{x} \varphi\right)-\mathrm{d} x \wedge \mathrm{d} \varphi .
\end{aligned}
$$

If $I=I\left(\alpha_{1}, \alpha_{2}, \alpha_{3}, \beta_{1}, \beta_{2}, \beta_{3}\right)$ is the ideal in the exterior algebra with basis $\left\{\mathrm{d} x, \mathrm{~d} t, \mathrm{~d} u, \mathrm{~d} u_{x}, \mathrm{~d} u_{x x}, \mathrm{~d} \varphi, \mathrm{d} \varphi_{x}, \mathrm{~d} \varphi_{x x}\right\}$, one can easily verify that the condition $\mathrm{d} I \subset I$ is satisfied. To prolong the ideal $I$ we add the even Lie superalgebra valued prolongation form

$$
\omega=\mathrm{d} \bar{y}+\mathrm{d} x F\left(u, u_{x}, u_{x x}, \varphi, \varphi_{x}, \varphi_{x x}\right)+\mathrm{d} t G\left(u, u_{x}, u_{x x}, \varphi, \varphi_{x}, \varphi_{x x}\right)
$$

where $F$ and $G$ are two even elements of the Lie superalgebra, and require that $\omega$ satisfy the condition $\mathrm{d} \omega+\mathrm{d} t \wedge \mathrm{d} t[G, F] \in I$. This condition leads to the overdetermined system of partial differential equations

$$
\begin{array}{cc}
F_{u}+G_{u_{x x}}=0 & F_{u_{x}}=F_{u_{x x}}=0 \\
F_{\varphi}+G_{\varphi_{x x}}=0 & F_{\varphi_{x}}=F_{\varphi_{x x}}=0 \\
\left(6 u u_{x}-3 \varphi \varphi_{x x}\right) F_{u}+\left(3 u \varphi_{x}+3 u_{x} \varphi\right) F_{\varphi}-u_{x} G_{u}-u_{x x} G_{u_{x}}-\varphi_{x} G_{\varphi}-\varphi_{x x} G_{\varphi_{x}}+[F, G] \\
=0 .
\end{array}
$$

We solved this system using a REDUCE package for computations in Lie superalgebras [7]. If we define $x_{i}$ with $i<0$ to be odd generators of the Lie superalgebra and $x_{i}$ with $i>0$ even generators, we find for $F$ and $G$

$$
\begin{aligned}
F=u \varphi x_{-2}+ & \varphi x_{-1}+x_{1}+u x_{2}+u^{2} x_{3} \\
G=\frac{1}{2}\left[-2 u \varphi x_{-6}\right. & -2 \varphi x_{-5}-2\left(-u \varphi_{x}+u_{x} \varphi\right) x_{-4}+2 \varphi_{x} x_{-3} \\
& -2\left(-3 u \varphi+\varphi_{x x}\right) x_{-1}-2\left(-4 u^{2} \varphi+u \varphi_{x x}-u_{x} \varphi_{x}+u_{x x} \varphi\right) x_{-2} \\
& -2\left(-3 u^{2}+u_{x x}+3 \varphi \varphi_{x}\right) x_{2}-2\left(-4 u^{3}+2 u u_{x x}+2 u \varphi \varphi_{x}-u_{x}^{2}\right) x_{3} \\
& \left.+2 x_{4}-2 u_{x} x_{5}-2 u x_{6}-u^{2} x_{7}+2 \varphi \varphi_{x} x_{8}\right]
\end{aligned}
$$


where we have defined

$$
\begin{array}{llll}
x_{5}=\left[x_{1}, x_{2}\right] & x_{6}=\left[x_{1}, x_{5}\right] & x_{7}=\left[x_{2}, x_{5}\right] & x_{8}=\left[x_{-1}, x_{-1}\right] \\
x_{-3}=\left[x_{-1}, x_{1}\right] & x_{-4}=\left[x_{-1}, x_{2}\right] & x_{-5}=\left[x_{-3}, x_{1}\right] & x_{-6}=\left[x_{-3}, x_{2}\right] .
\end{array}
$$

In addition we find that the following commutation relations hold

$$
\begin{array}{lll}
{\left[x_{-6}, x_{1}\right]=-\frac{1}{3}\left(\left[x_{-2}, x_{4}\right]-3 x_{-3}\right)} & {\left[x_{-4}, x_{-1}\right]=0} & {\left[x_{-4}, x_{1}\right]=0} \\
{\left[x_{-4}, x_{2}\right]=-x_{-2}} & {\left[x_{-2}, x_{-2}\right]=0} & {\left[x_{-2}, x_{-1}\right]=2 x_{3}} \\
{\left[x_{-2}, x_{1}\right]=0} & {\left[x_{-2}, x_{3}\right]=0} & {\left[x_{-2}, x_{2}\right]=0} \\
{\left[x_{-1}, x_{3}\right]=0} & {\left[x_{-1}, x_{4}\right]=-\left[x_{-5}, x_{1}\right]} & {\left[x_{-1}, x_{7}\right]=0} \\
{\left[x_{1}, x_{3}\right]=0} & {\left[x_{1}, x_{4}\right]=0} & {\left[x_{1}, x_{8}\right]=2 x_{5}} \\
{\left[x_{2}, x_{3}\right]=0} & {\left[x_{2}, x_{4}\right]=\left[x_{1}, x_{6}\right]} & {\left[x_{2}, x_{7}\right]=0} \\
{\left[x_{3}, x_{4}\right]=\frac{3}{2}\left(\left[x_{1}, x_{7}\right]-2 x_{5}\right)} & &
\end{array}
$$

\subsection{Realization of the prolongation algebra}

In this section we, as already mentioned, determine the structure of the prolongation algebra of the SKdV equation and give a realization of it, using the theory of KacMoody Lie superalgebras. Henceforth we denote this prolongation algebra, i.e. the Lie superalgebra generated by the even generators $x_{1}, \ldots, x_{8}$ and the odd generators $x_{-1}, \ldots, x_{-5}$ and subjected to relations (13) and (14), by MR. An important tool for determining the explicit structure is a grading. The following proposition is immediate.

Proposition 1. MR admits a $\mathbb{Z}$ grading

\begin{tabular}{lcccccccc} 
Generator & $x_{-6}$ & $x_{-5}$ & $x_{-4}$ & $x_{-3}$ & $x_{-2}$ & $x_{-1}$ & & \\
\cline { 1 - 6 } Degree & -1 & 3 & -3 & 1 & -5 & -1 & & \\
Generator & $x_{1}$ & $x_{2}$ & $x_{3}$ & $x_{4}$ & $x_{5}$ & $x_{6}$ & $x_{7}$ & $x_{8}$ \\
\hline Degree & 2 & -2 & -6 & 6 & 0 & 2 & -2 & -2
\end{tabular}

such that relations (13) and (14) are homogeneous.

Note that this $\mathbb{Z}$ grading is consistent with the $\mathbb{Z}_{2}$ grading of MR.

In [4] we showed how the Kac-Moody algebra $A_{1}^{(1)}$ and its realization can be used to find a realization of the prolongation algebra of the ordinary KdV equation. In this case the Kac-Moody Lie superalgebra $C^{(2)}(2)$ plays a similar role. We have the following proposition.

Proposition 2 (Kac [8], proposition 1.2). (a) $C^{(2)}(2)$ is the Lie superalgebra with even generators $H_{1}, H_{2}$, odd generators $E_{1}, E_{2}, F_{1}, F_{2}$ and defining relations $(i, j=1,2)$

$$
\begin{aligned}
& {\left[H_{i}, H_{j}\right]=0 \quad\left[E_{i}, F_{j}\right]=\delta_{i j} H_{i}} \\
& {\left[H_{i}, E_{j}\right]=a_{i j} E_{j} \quad\left[H_{i}, F_{j}\right]=-a_{i j} F_{j}} \\
& \left(\operatorname{ad} E_{i}\right)^{1-a_{i}} E_{j}=0 \quad\left(\operatorname{ad} F_{i}\right)^{1-a_{13}} F_{j}=0 \quad(i \neq j)
\end{aligned}
$$


where $a_{i i}=2$ and $a_{i j}=-2(i \neq j)$.

(b) If $C(2) \simeq A(1,0) \simeq \operatorname{osp}(2,2)$ is the (classical) eight-dimensional Lie superalgebra with Dynkin diagram $\otimes-\otimes, \sigma$ the automorphism of $C(2)$ of order 2 corresponding to interchanging the two vertices in the Dynkin diagram, with corresponding $\mathbb{Z}_{2}$ gradation $C(2)=C(2)_{\overline{0}} \oplus C(2)_{\overline{1}}$, and $C=\left\langle H_{1}+H_{2}\right\rangle$ the centre of $C^{(2)}(2)$, then

$$
C^{(2)}(2) / C \simeq \operatorname{Cov}(C(2), \sigma)=\bigoplus_{i \in \mathbb{Z}} \lambda^{i} C(2)_{i \bmod 2} \subset C(2) \otimes \mathbb{C}\left[\lambda, \lambda^{-1}\right] .
$$

where $\lambda$ is an indeterminate.

Table 1. Commutator table of $C(2)_{\overline{0}} \oplus C(2)_{\bar{j}}$.

\begin{tabular}{llllccccc}
\hline & $h_{0}$ & $e_{1}$ & $e_{2}$ & $f_{-1}$ & $f_{-2}$ & $v_{0}$ & $v_{1}$ & $v_{-1}$ \\
\hline$h_{0}$ & 0 & $e_{1}$ & $2 e_{2}$ & $-f_{-1}$ & $-2 f_{-2}$ & 0 & $v_{1}$ & $-v_{-1}$ \\
$e_{1}$ & & $2 e_{2}$ & 0 & $h_{0}$ & $f_{-1}$ & $-v_{1}$ & 0 & $-v_{0}$ \\
$e_{2}$ & & & 0 & $-e_{1}$ & $h_{0}$ & 0 & 0 & $v_{1}$ \\
$f_{-1}$ & & & & $-2 f_{-2}$ & 0 & $v_{-1}$ & $-v_{0}$ & 0 \\
$f_{-2}$ & & & & & 0 & 0 & $v_{-1}$ & 0 \\
$v_{0}$ & & & & & & 0 & $e_{1}$ & $-f_{-1}$ \\
$v_{1}$ & & & & & & & $-2 e_{2}$ & $h_{0}$ \\
$v_{-1}$ & & & & & & & & $2 f_{-2}$ \\
\hline
\end{tabular}

Working out the construction of proposition 2 , we see that $C(2)_{\overline{0}}=$ $\left\langle h_{0}, e_{1}, e_{2}, f_{-1}, f_{-2}\right\rangle$ and $C(2)_{\overline{1}}=\left\langle v_{0}, v_{1}, v_{-1}\right\rangle$. The even and odd elements of $C(2)_{\overline{0}}$ and $C(2)_{\overline{1}}$ are just those with even and odd indices, respectively. The commutator table of $C(2)_{\overline{0}} \oplus C(2)_{\overline{1}}$ can be found in table 1 . Note that $C(2)_{\overline{0}}$ constitutes a $B(0,1) \simeq \operatorname{osp}(1,2),\left\langle h_{0}, e_{2}, f_{-2}\right\rangle$ a $s l(2)$ and $C(2)_{\overline{1}}$ is a $C(2)_{\overline{0}}$ module.

The isomorphism $C^{(2)}(2) / C \rightarrow \bigoplus_{i \in \mathbb{Z}} \lambda^{i} C(2)_{i \bmod 2}$ is given explicitly by

$$
\begin{array}{lll}
E_{1} \mapsto e_{1} & F_{1} \mapsto f_{-1} & H_{1} \mapsto h_{0} \\
E_{2} \mapsto \lambda v_{-1} & F_{2} \mapsto \lambda^{-1} v_{1} . &
\end{array}
$$

As in the ordinary case we are only interested in the positive part of $C^{(2)}(2)$. Equating to zero $F_{2}$ yields the following.

Proposition 3. The Lie superalgebra with even generator $H_{1}$, odd generators $E_{1}, F_{1}, E_{2}$ and defining relations

$$
\begin{array}{ll}
{\left[E_{1}, F_{1}\right]=H_{1}} & {\left[H_{1}, E_{2}\right]=-2 E_{2}} \\
{\left[H_{1}, E_{1}\right]=2 E_{1}} & {\left[H_{1}, F_{1}\right]=-2 F_{1}} \\
\left(\operatorname{ad} E_{1}\right)^{3} E_{2}=\left(\operatorname{ad} E_{2}\right)^{3} E_{1}=0 &
\end{array}
$$

is isomorphic to the algebra $\operatorname{Cov}^{+}(C(2), \sigma)=\bigoplus_{i \in \mathbb{N}} \lambda^{i} C(2)_{i \bmod 2}$, the isomorphism being given by

$$
H_{1} \mapsto h_{0} \quad E_{1} \mapsto e_{1} \quad F_{1} \mapsto f_{-1} \quad E_{2} \mapsto \lambda v_{-1}
$$


Table 2. Commutator table of MR.

\begin{tabular}{|c|c|c|c|c|c|c|c|c|c|}
\hline & $x-16$ & $x-10$ & $x-8$ & $x-6$ & $x-5$ & $x_{-4}$ & $x-3$ & $x-2$ & $x_{-1}$ \\
\hline$x-16$ & $4 x_{11}-x_{16}$ & $x_{20}$ & $-2 x_{10}$ & $-\left(x_{14}+2 x_{9}\right)$ & $x_{13}-2 x_{11}$ & 0 & $-x_{10}$ & 0 & $-\left(x_{14}+2 x_{9}\right)$ \\
\hline$x-10$ & & $x_{19}+x_{17}$ & $x_{12}$ & $x_{13}$ & $x_{18}-x_{20}$ & 0 & 0 & 0 & $x_{13}$ \\
\hline$x-8$ & & & $x_{14}$ & 0 & $x_{10}$ & 0 & $-x_{9}$ & 0 & 0 \\
\hline$x-6$ & & & & $x_{7}$ & $x_{9}$ & 0 & $-x_{5}$ & 0 & $x_{7}$ \\
\hline$x-5$ & & & & & $x_{11}-x_{13}$ & 0 & 0 & 0 & $x_{9}$ \\
\hline$x-4$ & & & & & & $2 x_{3}$ & 0 & 0 & 0 \\
\hline$x-3$ & & & & & & & $x_{6}-x_{9}$ & 0 & $-x_{5}$ \\
\hline$x-2$ & & & & & & & & 0 & $2 x_{3}$ \\
\hline$x-1$ & & & & & & & & & $x_{B}$ \\
\hline
\end{tabular}

\begin{tabular}{|c|c|c|c|c|c|c|c|c|c|}
\hline & $x_{1}$ & $x_{2}$ & $x_{3}$ & $x_{4}$ & $x_{5}$ & $x_{6}$ & $x_{?}$ & $x_{8}$ & $x_{9}$ \\
\hline$x-16$ & $x-7$ & $-2 x-8$ & 0 & $-x-9$ & $-x-16$ & $x-11$ & $-4 x-8$ & $-4 x-8$ & $3 x-11-2 x-7$ \\
\hline$x-10$ & $x-9$ & $x-11$ & 0 & $x-12$ & $x-14-x-10$ & $-\left(x_{-9}+x_{-20}\right)$ & $2 x-11$ & $2 x-11$ & $-(x-9+x-20)$ \\
\hline$x-8$ & $x-5$ & 0 & 0 & $x-10$ & $x-8$ & $x-16$ & 0 & 0 & $-(2 x-5+x-16)$ \\
\hline$x-6$ & $x-3$ & 0 & 0 & $x-7$ & $x-6$ & $x-8-2 x-3$ & 0 & 0 & $-x-8$ \\
\hline$x-5$ & $-x-7$ & $x-8$ & 0 & $x-9$ & $x-5+x-16$ & $x-7-x-11$ & $2 x-8$ & $2 x-8$ & $x-7-x-11$ \\
\hline$x-4$ & 0 & $-x-2$ & 0 & 0 & 0 & 0 & 0 & 0 & 0 \\
\hline$x-3$ & $x_{-5}$ & $x-6$ & 0 & $x-10$ & $x_{-8}-x_{-3}$ & $x_{-5}+x_{-16}$ & $2 x-6$ & $2 x-6$ & $x_{-5}+x-16$ \\
\hline$x-2$ & 0 & 0 & 0 & 0 & 0 & 0 & 0 & 0 & 0 \\
\hline$x-1$ & $x-3$ & $x-4$ & 0 & $x_{-7}$ & $x_{-6}$ & $x_{-8}-2 x-3$ & 0 & 0 & $-x-8$ \\
\hline$x_{1}$ & 0 & $x_{5}$ & 0 & $\mathbf{0}$ & $x_{6}$ & $x_{10}$ & $2 x_{5}$ & $2 x_{5}$ & $x_{10}$ \\
\hline$x_{2}$ & & 0 & 0 & $x_{10}$ & $x_{7}$ & $2 x_{5}$ & 0 & 0 & 0 \\
\hline$x_{3}$ & & & 0 & 0 & 0 & 0 & 0 & 0 & 0 \\
\hline$x_{4}$ & & & & 0 & $x_{11}$ & $x_{12}$ & $-2 x_{10}$ & $-2 x_{10}$ & $x_{12}$ \\
\hline$x_{5}$ & & & & & 0 & $x_{14}+2 x_{6}$ & $-2 x_{7}$ & $-2 x_{7}$ & $x_{14}$ \\
\hline$x_{6}$ & & & & & & 0 & $-4 x_{5}$ & $-4 x_{5}$ & $-2 x_{10}$ \\
\hline$x_{7}$ & & & & & & & 0 & 0 & 0 \\
\hline$x_{8}$ & & & & & & & & 0 & 0 \\
\hline$x_{9}$ & & & & & & & & & 0 \\
\hline
\end{tabular}

With the help of the previously mentioned package we computed part of the commutator table of MR. The part of it relevant for finding a realization of MR can be found in table 2. In principle this table can be checked by hand using the graded Jacobi identity

$$
[x,[y, z]]=[[x, y], z]+(-1)^{|x||y|}[y,[x, z]]
$$

Using this table and the grading of proposition 1 one can easily find a subalgebra of MR isomorphic to $C(2)_{\overline{0}}$, namely

$$
\begin{array}{lll}
f_{-2}=-\frac{1}{2} x_{7} & f_{-1}=x_{-6} & h_{0}=x_{5} \\
e_{1}=-\frac{1}{2}\left(2 x_{-3}-x_{-8}\right) & e_{2}=\frac{1}{8}\left(x_{14}+4 x_{6}\right) . &
\end{array}
$$

From this it is not very difficult to find a set $H_{1}, E_{1}, F_{1}, E_{2}$ satisfying relations (15), we can namely take $H_{1}=h_{0}, E_{1}=e_{1}, F_{1}=f_{-1}$ and $E_{2}=x_{-8}$.

Moreover we see that $\mathrm{MR}$ contains a eight-dimensional subalgebra $R_{\mathrm{MR}}=$ $\left\langle c_{1}, c_{2}, c_{3}, c_{4}, r_{1}, r_{2}, r_{3}, r_{4}\right\rangle$ commuting with $H_{1}, E_{1}, F_{1}, E_{2}$, where 
Table 2. (Continued)

\begin{tabular}{|c|c|c|c|c|c|}
\hline & $x_{10}$ & $x_{11}$ & $x_{13}$ & $x_{14}$ & $x_{16}$ \\
\hline$x-16$ & $x-14-2 x-10$ & $x-20$ & $2 x-9+3 x-20$ & $-4 x-11$ & $4 x-20$ \\
\hline$x-10$ & $x-13-x-21$ & $x-18-x-12$ & $x-18-x-12$ & $2 x-20$ & $2 x-18$ \\
\hline$x-8$ & $-x-11$ & $x_{-14}-2 x-10$ & $-x_{-14}$ & 0 & 0 \\
\hline$x-6$ & $2 x-5+x-16$ & $x-11-2 x-7$ & $-x-11$ & 0 & 0 \\
\hline$x-5$ & $x-10-x-14$ & $-(x-9+x-20)$ & $-(x-9+x-20)$ & $2 x-11$ & $-2 x-20$ \\
\hline$x-4$ & 0 & 0 & 0 & 0 & 0 \\
\hline$x-3$ & $x_{-7}-x-11$ & $x_{-14}-x-10$ & $x_{-14}-x-10$ & $-2\left(2 x_{-5}+x_{-16}\right)$ & $2 x-14$ \\
\hline$x-2$ & 0 & 0 & 0 & 0 & 0 \\
\hline$x-1$ & $2 x-5+x-16$ & $x-11-2 x-7$ & $-x-11$ & 0 & 0 \\
\hline$x_{1}$ & $-x_{11}$ & $x_{12}$ & $x_{12}$ & $-2 x_{10}$ & $2 x_{12}$ \\
\hline$x_{2}$ & $-x_{14}$ & $-2 x_{10}$ & 0 & 0 & 0 \\
\hline$x_{3}$ & 0 & 0 & 0 & 0 & 0 \\
\hline$x_{4}$ & $x_{18}$ & $x_{15}$ & $x_{15}$ & $-2 x_{12}$ & $2 x_{15}$ \\
\hline$x_{5}$ & 0 & $2 x_{11}-x_{16}$ & $-x_{16}$ & $-2 x_{14}$ & $-2 x_{16}$ \\
\hline$x_{6}$ & $2 x_{11}-x_{16}$ & 0 & $-2 x_{12}$ & $4 x_{10}$ & $-4 x_{12}$ \\
\hline$x_{7}$ & $-2 x_{14}$ & $-4 x_{10}$ & 0 & 0 & 0 \\
\hline$x_{8}$ & $-2 x_{14}$ & $-4 x_{10}$ & 0 & 0 & 0 \\
\hline$x_{9}$ & $-x_{16}$ & $2 x_{12}$ & 0 & 0 & 0 \\
\hline$x_{10}$ & 0 & $2 x_{18}-x_{22}$ & $-x_{22}$ & $-2 x_{16}$ & $-2 x_{22}$ \\
\hline$x_{11}$ & & 0 & $-2 x_{15}$ & $4 x_{12}$ & $-4 x_{15}$ \\
\hline$x_{13}$ & & & 0 & 0 & 0 \\
\hline$x_{14}$ & & & & 0 & 0 \\
\hline$x_{16}$ & & & & & 0 \\
\hline
\end{tabular}

$$
\begin{array}{ll}
c_{1}=x_{3} & r_{1}=2 x_{2}-x_{7} r_{3}=x_{-2} \\
c_{2}=x_{7}-x_{8} & r_{2}=x_{-4} \\
c_{3}=2 x_{1}+x_{6}+x_{9}+x_{14} & r_{4}=x_{-1}-x_{-6} \\
c_{4}=2 x_{4}+x_{11}+x_{13}-x_{16} . &
\end{array}
$$

One can easily verify that $c_{1}, c_{2}, c_{3}, c_{4}$ are central elements of MR. $r_{1}, r_{2}, r_{3}, r_{4}$ satisfy the relations

$\left[r_{1}, r_{2}\right]=2 r_{3} \quad\left[r_{1}, r_{4}\right]=-2 r_{2} \quad\left[r_{2}, r_{2}\right]=\left[r_{3}, r_{4}\right]=2 c_{1} \quad\left[r_{4}, r_{4}\right]=-c_{2}$

all other commutators being zero. We can now easily prove the following theorem.

Theorem 1. MR is isomorphic to the algebra $R_{\mathrm{MR}} \times \mathrm{Cov}^{+}(C(2), \sigma)$.

Proof. Let $L\left(H_{1}, E_{1}, F_{1}, E_{2}, c_{1}, \ldots, c_{4}, r_{1}, \ldots, r_{4}\right)$ be the free Lie superalgebra on even generators $H_{1}, c_{1}, \ldots, c_{4}, r_{1}$ and odd generators $E_{1}, F_{1}, E_{2}, r_{2}, r_{3}, r_{4}$ and consider the Lie superalgebra morphism $\phi: L\left(H_{1}, E_{1}, F_{1}, E_{2}, c_{1}, \ldots, c_{4}, r_{1}, \ldots, r_{4}\right) \rightarrow$ MR given by

$$
\begin{array}{llll}
H_{1} \mapsto x_{5} & E_{1} \mapsto-\frac{1}{2}\left(2 x_{-3}-x_{-8}\right) & F_{1} \mapsto x_{-6} & E_{2} \mapsto x_{-8} \\
c_{1} \mapsto x_{3} & c_{3} \mapsto 2 x_{1}+x_{6}+x_{9}+x_{14} & r_{1} \mapsto 2 x_{2}-x_{7} & r_{3} \mapsto x_{-2} \\
c_{2} \mapsto x_{7}-x_{8} & c_{4} \mapsto 2 x_{4}+x_{11}+x_{13}-x_{16} & r_{2} \mapsto x_{-4} & r_{4} \mapsto x_{-1}-x_{-6} .
\end{array}
$$

As we have already seen $\phi$ leaves invariant relations (15) and (16), hence there is a Lie superalgebra morphism $\phi^{\prime}: R_{\mathrm{MR}} \times \mathrm{Cov}^{+}(C(2), \sigma) \rightarrow \mathrm{MR}$. On the other hand the 
Lie superalgebra morphism $\psi: L\left(x_{1}, \ldots, x_{8}, x_{-1}, \ldots, x_{-6}\right) \rightarrow R_{\mathrm{MR}} \times \mathrm{Cov}^{+}(C(2), \sigma)$ given by

$$
\begin{array}{ll}
x_{1} \mapsto \frac{1}{4}\left(2 c_{3}-4 e_{2}-\lambda^{2} f_{-2}+2 \lambda v_{0}\right) & x_{2} \mapsto-\frac{1}{2}\left(2 f_{-2}-r_{1}\right) \\
x_{3} \mapsto c_{1} & x_{4} \mapsto \frac{1}{4}\left(2 c_{4}+4 \lambda^{2} e_{2}+\lambda^{4} f_{-2}-2 \lambda^{3} v_{0}\right) \\
x_{5} \mapsto h_{0} & x_{6} \mapsto \frac{1}{2}\left(4 e_{2}-\lambda^{2} f_{-2}\right) \\
x_{7} \mapsto-2 f_{-2} & x_{8} \mapsto-\left(c_{2}+2 f_{-2}\right) \\
x_{-1} \mapsto r_{4}+f_{-1} & x_{-2} \mapsto r_{3} \\
x_{-3} \mapsto-\frac{1}{2}\left(2 e_{1}-\lambda v_{-1}\right) & x_{-4} \mapsto r_{2} \\
x_{-5} \mapsto \frac{1}{2}\left(2 \lambda v_{1}+\lambda^{2} f_{-1}\right) & x_{-6} \mapsto f_{-1}
\end{array}
$$

preserves relations (13) and (14), hence there exists a Lie superalgebra morphism $\psi^{\prime}: \mathrm{MR} \rightarrow R_{\mathrm{MR}} \times \mathrm{Cov}^{+}(C(2), \sigma) . \phi^{\prime}$ and $\psi^{\prime}$ are easily seen to be each other's inverse.

\section{Auto-Bäcklund transformation, superposition principle and relationship with known solution methods for the skdV equation}

In this section we derive an auto-Bäcklund transformation for the SKdV equation from the prolongation structure and give a formula from which it is possible to derive a superposition principle for solutions of the SKdV equation. Moreover we give the relationship between the prolongation method and known solution methods for the SKdV equation, such as the super-Miura transformation and the super-Gardner transformation.

For this we need a (graded) vector field representation of $C(2)$, in order to be able to retrieve $F^{0}, F^{1}, G^{0}, G^{1}$ from $F$ and $G$, respectively, using formulae (8), (11) and (12). In the sequel we use the following vector field representation of $C(2)$, which can easily be checked using formula (1) and table 1.

$$
\begin{aligned}
& f_{-2}=\lambda_{1}^{-1} \lambda_{2}^{-1} \mu_{2}^{-2}\left(-y^{1-\mu_{2}} \partial_{y}+\left(\mu_{1}-1\right) y^{-\mu_{2}} \xi \partial_{\xi}\right) \\
& f_{-1}=\lambda_{2}^{-1} \mu_{2}^{-1} y^{\mu_{1}-\mu_{2}} \xi \partial_{y}+\lambda_{1}^{-1} \mu_{2}^{-1} y^{1-\mu_{1}} \partial_{\xi} \\
& h_{0}=2 \mu_{2}^{-1} y \partial_{y}+\left(1-2 \mu_{1} \mu_{2}^{-1}+2 \mu_{2}^{-1}\right) \xi \partial_{\xi} \\
& e_{1}=\lambda_{1} y^{\mu_{1}} \xi \partial_{y}+\lambda_{2} y^{1-\mu_{1}+\mu_{2}} \partial_{\xi} \\
& e_{2}=\lambda_{1} \lambda_{2}\left(y^{1+\mu_{2}} \partial_{y}+\left(1-\mu_{1}+\mu_{2}\right) y^{\mu_{2}} \xi \partial_{\xi}\right) \\
& v_{-1}=\lambda_{2}^{-1} \mu_{2}^{-1} y^{\mu_{1}-\mu_{2}} \xi \partial_{y}-\lambda_{1}^{-1} \mu_{2}^{-1} y^{1-\mu_{1}} \partial_{\xi} \\
& v_{0}=-\xi \partial_{\xi} \\
& v_{1}=-\lambda_{1} y^{\mu_{1}} \xi \partial_{y}+\lambda_{2} y^{1-\mu_{1}+\mu_{2}} \partial_{\xi} .
\end{aligned}
$$

Equating $c_{1}, \ldots, c_{4}, r_{1}, \ldots, r_{4}$ to zero, substituting the representation (18) with $\lambda_{1}=$ $\lambda_{2}=\mu_{1}=\mu_{2}=1$ into the isomorphism (17), replacing $\lambda$ by $2 \lambda$ (to avoid denominators) and finally taking $y^{0}=y$ and $y^{1}=\xi$, we find from equations (11) and (12)

$$
F^{0}=\varphi \xi-y^{2}+\lambda^{2}+u
$$




$$
\begin{gathered}
F^{1}=\varphi-(y+\lambda) \xi \\
G^{0}=-\left(\varphi_{x x} \xi+(y-\lambda) \varphi_{x} \xi+2\left(-y \lambda+\lambda^{2}-u\right) \varphi \xi+\varphi \varphi_{x}-4 y^{2} \lambda^{2}+2 y^{2} u\right. \\
\left.+2 y u_{x}+4 \lambda^{4}+2 \lambda^{2} u-2 u^{2}+u_{x x}\right) \\
G^{1}=\left(4 y \lambda^{2}-2 y u+4 \lambda^{3}-u_{x}\right) \xi-\varphi_{x x}-(y+\lambda) \varphi_{x}+2\left(-y \lambda-\lambda^{2}+u\right) \varphi .
\end{gathered}
$$

On a solution manifold of the prolonged ideal we have, as in the ordinary case

$$
y_{x}=-F^{0} \quad y_{t}=-G^{0} \quad \xi_{x}=-F^{1} \quad \xi_{t}=-G^{1} .
$$

One readily verifies the integrability conditions $y_{x t}=y_{t x}$ for $y$ and $\xi_{x t}=\xi_{t x}$ for $\xi$ to be satisfied if and only if $u$ and $\varphi$ satisfy the SKdV equation.

If $u, \varphi$ is a solution to equations (9) and (10), we can find a Bäcklund transformation by requiring $\tilde{u}=\tilde{u}\left(u^{(2)}, \varphi^{(2)}, y, \xi\right)$ and $\tilde{\varphi}=\tilde{\varphi}\left(u^{(2)}, \varphi^{(2)}, y, \xi\right)$ to be another solution to the SKdV equation. Another way of posing this is to require that the set of forms

$$
\begin{aligned}
& \tilde{\alpha}_{1}=\mathrm{d} t \wedge \mathrm{d} \tilde{u}+\mathrm{d} x \wedge \mathrm{d} t \tilde{u}_{x} \\
& \tilde{\alpha}_{2}=\mathrm{d} t \wedge \mathrm{d} \tilde{u}_{x}+\mathrm{d} x \wedge \mathrm{d} t \tilde{u}_{x x} \\
& \tilde{\alpha}_{3}=\mathrm{d} t \wedge \mathrm{d} \tilde{u}_{x x}+d x \wedge \mathrm{d} t\left(6 \tilde{u} \tilde{u}_{x}-3 \tilde{\varphi} \tilde{\varphi}_{x x}\right)-\mathrm{d} x \wedge \mathrm{d} \tilde{u} \\
& \tilde{\beta}_{1}=\mathrm{d} t \wedge \mathrm{d} \tilde{\varphi}+\mathrm{d} x \wedge \mathrm{d} t \tilde{\varphi}_{x} \\
& \tilde{\beta}_{2}=\mathrm{d} t \wedge \mathrm{d} \tilde{\varphi}_{x}+\mathrm{d} x \wedge \mathrm{d} t \tilde{\varphi}_{x x} \\
& \tilde{\beta}_{3}=\mathrm{d} t \wedge \mathrm{d} \tilde{\varphi}_{x x}+\mathrm{d} x \wedge \mathrm{d} t\left(3 \tilde{u} \tilde{\varphi}_{x}+3 \tilde{u}_{x} \tilde{\varphi}\right)-\mathrm{d} x \wedge \mathrm{d} \tilde{\varphi}
\end{aligned}
$$

be elements of the prolonged ideal $I\left(\alpha_{1}, \ldots, \alpha_{4}, \beta_{1}, \ldots, \beta_{4}, \omega^{0}, \omega^{1}\right)$. By a rather straightforward, but tedious computation we discover that

$$
\begin{aligned}
& \tilde{u}=-2 \varphi \xi+2 y^{2}-2 \lambda^{2}-u=u+2 y_{x} \\
& \tilde{\varphi}= \pm(-\varphi+2(y+\lambda) \xi)= \pm\left(\varphi+2 \xi_{x}\right)
\end{aligned}
$$

is always another solution to the SKdV equation.

Solving $u$ from the equation $y_{x}=-F^{0}$ and $\varphi$ from $\xi_{x}=-F^{1}$ we find

$$
\begin{aligned}
& u=-y_{x}+y^{2}-\lambda^{2}-\varphi \xi=-y_{x}+y^{2}-\lambda^{2}-\xi \xi_{x} \\
& \varphi=-\xi_{x}+(y+\lambda) \xi
\end{aligned}
$$

Substituting (22) and (23) into the equations $y_{t}=-G^{0}$ and $\xi_{t}=-G^{1}$, we find that $y$ and $\xi$ have to satisfy the equations

$$
\begin{aligned}
& y_{t}=-y_{x x x}+6\left(y^{2}-\lambda^{2}\right) y_{x}-3 \xi\left((y+\lambda) \xi_{x}\right)_{x} \\
& \xi_{t}=-\xi_{x x x}+3(y+\lambda)((y-\lambda) \xi)_{x} .
\end{aligned}
$$

Notice that the transformation $(y, \xi, \lambda) \mapsto(-y, \pm \xi,-\lambda)$ leaves invariant equations (24) and (25). This gives us another way of obtaining the Bäcklund transformation (20) 
and (21). Namely, the triple $(-y,-\xi,-\lambda)$ can be associated with a solution $\tilde{u}, \tilde{\varphi}$ of the SKdV equation. Hence we have

$$
\begin{aligned}
& y_{x}=-\varphi \xi+y^{2}-\lambda^{2}-u \\
& -y_{x}=\tilde{\varphi} \xi+y^{2}-\lambda^{2}-\tilde{u} \\
& \xi_{x}=-\varphi+(y+\lambda) \xi \\
& -\xi_{x}=-\tilde{\varphi}+(y+\lambda) \xi .
\end{aligned}
$$

Now subtracting equations (28) and (29) leads to the relation $\tilde{\varphi}=\varphi+2 \xi_{x}$ and subtracting equations $(26)$ and $(27)$ gives $\tilde{u}=u+2 y_{x}+(\varphi+\tilde{\varphi}) \xi=u+2 y_{x}+2\left(\varphi+\xi_{x}\right) \xi=$ $u+2 y_{x}+2(y+\lambda) \xi^{2}=u+2 y_{x}$.

As in [1] we can also use the prolongation structure to derive a superposition principle for solutions of the SKdV equation. We can find it by entering the forms $\tilde{\alpha}_{1}, \ldots, \tilde{\beta}_{3}$ with the ansatz $\tilde{u}=\tilde{u}\left(u, \varphi, y_{1}, \xi_{1}, y_{2}, \xi_{2}\right)$, where $y_{1}=y_{1}\left(\lambda_{1}\right), \xi_{1}=\xi_{1}\left(\lambda_{1}\right)$ and $y_{2}=y_{2}\left(\lambda_{2}\right), \xi_{2}=\xi_{2}\left(\lambda_{2}\right)$. In this way we find that

$$
\begin{aligned}
\tilde{u}=u+2 & \frac{\left(\lambda_{1}^{2}-\lambda_{2}^{2}\right)\left(\lambda_{1}^{2}-\lambda_{2}^{2}-y_{1}^{2}+y_{2}^{2}\right)}{\left(y_{1}-y_{2}\right)^{2}}+2 \frac{\lambda_{1}+\lambda_{2}}{y_{1}-y_{2}}\left(\varphi \xi_{2}-\varphi \xi_{1}\right) \\
& +4\left(\frac{\lambda_{1}^{2} y_{2}-\lambda_{2}^{2} y_{1}-\left(\lambda_{1}+\lambda_{2}\right)^{2}\left(\lambda_{1}-\lambda_{2}\right)}{\left(y_{1}-y_{2}\right)^{2}}-\frac{\left(\lambda_{1}^{2}-\lambda_{2}^{2}\right)^{2}}{\left(y_{1}-y_{2}\right)^{3}}-\frac{\lambda_{1} \lambda_{2}}{y_{1}-y_{2}}\right) \xi_{1} \xi_{2}
\end{aligned}
$$

$\tilde{\varphi}=\varphi-2\left(\lambda_{1}+\lambda_{2}\right)\left(\frac{\lambda_{1}^{2}-\lambda_{2}^{2}}{\left(y_{1}-y_{2}\right)^{2}}\left(\xi_{1}-\xi_{2}\right)+\frac{\lambda_{1}-y_{2}}{y_{1}-y_{2}} \xi_{1}-\frac{\lambda_{2}-y_{1}}{y_{1}-y_{2}} \xi_{2}\right)$

is also another solution to the SKdV equation. Note that the first two terms of $(30)$ constitute the superposition principle for the ordinary KdV equation as found by Wahlquist and Estabrook. If we use formulae (20) and (21) to express $y_{1}, \xi_{1}$ and $y_{2}, \xi_{2}$ in $u, \varphi$ and $u_{1}, \varphi_{1}$ and $u_{2}, \varphi_{2}$, respectively, equations (30) and (31) lead to a superposition principle for solutions of the SKdV equation. At this point, however, we do not perform this computation explicitly, because solving equations (20) and (21) gives rise to a cubic equation for $y_{1}$ and $y_{2}$.

To give the relationship with known solution methods for the SKdV equation we notice that for $\lambda=0$ equations (24) and (25) exactly are the super-modified $\mathrm{KdV}$ (SMKdV) equation, whereas for $\lambda=0$ equations (22) and (23) represent the superMiura transformation for the SKdV equation, which can be used to derive the linear problem for the SKdV equation ( $\mathrm{cf}[9]$ ).

More generally, by substituting $\lambda=1 / 2 \epsilon, y=\epsilon w+\lambda$ and $\xi=\epsilon \sigma$, equations (24) and (25) transform into the super-Gardner equation

$$
\begin{aligned}
& w_{t}=-w_{x x x}+6 w w_{x}-3 \sigma \sigma_{x x}+\epsilon^{2}\left(6 w^{2} w_{x}-3 \sigma\left(\sigma_{x} w\right)_{x}\right) \\
& \sigma_{t}=-\sigma_{x x x}+3(\sigma w)_{x}+3 \epsilon^{2} w(w \sigma)_{x}
\end{aligned}
$$

With the above substitution equations (22) and (23) transform into the super-Gardner transformation, which can be used to show the existence of an infinite number of conservation laws for the SKdV equation (cf [9]). In fact this boils down to the fact that $y_{t}$ can be written as a total derivative. 


\section{Conclusions}

In this paper we have shown how the prolongation method of Wahlquist and Estabrook can successfully be generalized to the case of supersymmetric equations. Application of this generalization to the SKdV equation of Manin-Radul leads to the rediscovery of known solution methods for the equation, but moreover provides us with a very systematic way to derive an auto-Bäcklund transformation and a superposition principle.

In [10], using the AKNS method, a relationship was shown between the Lie superalgebra $\operatorname{osp}(1,2)$ and the super KdV equation of Kupershmidt [11]. Using super prolongation theory, we have shown a similar relationship between $\operatorname{osp}(1,2)=B(0,1)$ and the SKdV equation of Manin-Radul (namely if we take $\lambda=0$ ). Moreover, for $\lambda \neq 0$ we have found a relationship with the Kac-Moody Lie superalgebra $C^{(2)}(2)$. It is easy to see that $C^{(2)}(2)$ contains $A_{1}^{(1)}$ as an (even) subalgebra, the positive part of which is exactly the main part of the prolongation algebra of the ordinary $\mathrm{KdV}$ equation. We recall that the $\tau$-function approach of Date et al [12] relates $A_{1}^{(1)}$ to the $\mathrm{KdV}$ equation in another way. It would be interesting to know if a similar construction relates $C^{(2)}(2)$ to the SKdV equation.

\section{References}

[1] Wahlquist W D and Estabrook F B 1975 J. Math. Phys. 16 1-7

[2] Estabrook F B 1989 Partially Integrable Nonlinear Evolution Equations and Their Physical Applications (Proc. NATO Adv. Study Inst.) (Dordrecht: Kluwer)

[3] van Eck H N 1983 Proc. Kon. Ned. Akad. Wetensch. A 86 149-63

[4] Roelofs G H M and Martini R 1990 J. Phys. A.: Math. Gen. 23 1877-84

[5] Kostant B 1977 Differential Geometrical Methods in Mathematical Physics (Berlin: Springer) pp 177-306

[6] Cartan E 1971 Les Systemes Différentiels Extérieurs et Leurs Applications Géometrique (Paris: Hermann)

[7] Roelofs G H M 1990 A Lie superalgebra package Memorandum University of Twente

[8] Kac V G 1978 Adv. Math. 30 85-136

[9] Mathieu P 1988 J. Math. Phys. 29 2499-506

[10] Gürses M and Öguz O 1986 Lett. Math. Phys. 11235

[11] Kupershmidt B A 1984 Phys. Lett. 102A 213-5

[12] Date E, Jimbo M, Kashiwara M, and Miwa T 1983 Classical Theory and Quantum Theory (Proc. RIMS Symp. on Non-linear Integrable Systems) (Singapore: World Scientific) 